\title{
Preparing for the next vintage in IE
}

\author{
Paola Anna Erba, MD, PhD, ${ }^{\mathrm{a}, \mathrm{b}}$ and Ora Israel ${ }^{\mathrm{c}}$ \\ a Regional Center of Nuclear Medicine, Department of Translational Research and New \\ Technology in Medicine, University of Pisa, and Azienda Ospedaliero Universitaria Pisana, \\ Pisa, Italy \\ b Department of Nuclear Medicine and Molecular Imaging, Medical Imaging Centre, University \\ Medical Center Groningen, Groningen, The Netherlands \\ c Rappaport, Faculty of Medicine Technion, Haifa, Israel
}

Received Jun 30, 2021; accepted Jun 30, 2021

doi: $10.1007 / \mathrm{s} 12350-021-02746-z$

See related article, pp. 2188-2194

Wine experts, writers, collectors and makers consider wine's vintage as a sign of excellence. In the field of multimodality imaging for diagnosis and management of patients with IE the vintage year was certainly 2015, the year when the latest version of the European Society of Cardiology (ESC) guidelines on Prevention, Diagnosis and Treatment of Infective Endocarditis (IE) ${ }^{1}$ were released.

In August 2015, with a backbone of brilliant preliminary results and strong intuitive clues, a group of pioneers grabbed these opportunities and generated the concept of expanding the armamentarium of diagnostic imaging with tools that may assist in cases when the standard procedures, i.e. transthoracic and transesophageal echocardiography are failing to provide a clear diagnosis, of particular significance in high risk patients with suspected prosthetic valve endocarditis. This successful initiative has been followed by a dynamic interplay of the imaging and clinical community who took the challenge of designing the necessary clinical studies that would generate relevant results to provide evidence to support and even expand the application of the multimodality approach into routine clinical practice. It took a few more years, but recently

Reprint requests: Paola Anna Erba, MD, PhD, Regional Center of Nuclear Medicine, Department of Translational Research and New Technology in Medicine, University of Pisa, and Azienda Ospedaliero Universitaria Pisana, Via Savi 10, 56126 Pisa, Italy; paola.erba@unipi.it

J Nucl Cardiol 2022;29:2195-6.

$1071-3581 / \$ 34.00$

Copyright (c) 2021 American Society of Nuclear Cardiology. the US community has also embraced a similar strategy. This has been proven by the American Heart Association (AHA) 2020 guidelines for the management of patients with valvular heart disease ${ }^{2}$ which also recognize the value of the multimodality approach and the importance of team work in the assessment of patients with IE.

Looking back to the development of the ESC guideline we have to admit that it took a lot of courage to endorse this very ambitious project. This approach gave it the power to lead a positive change. Inviting imaging experts to join the IE Team with a newly created role while most of the others players at the table had clear functions, established years ago, was not an easy step. However, changes bring opportunities! Within the IE Team, imagers have increased their and their counterparts confidence. Through an open and fair discussion, which also included acknowledgment of each modality limits, we took the challenge of rethinking our technology and workflow. We have attempted, and in large succeeded, to optimize the imaging protocol including image reconstruction methods for more reliable study interpretation, as well as to improve our skills in reading and reporting our data. ${ }^{3,4}$ In other words, we took the challenge to grow and mature, both as individual specialists but also as a speciality.

Over time a clear definition of the subgroups of patients most likely to benefit from the Multimodality, Multitracer and Multidisciplinary approach has emerged. If multimodality imaging is currently playing a significant role in the diagnostic algorithm of IE, CIED and LVAD infections, ${ }^{5}$ this does not necessarily spell the end of the scientific research in the field of this clinical application. On the contrary, as the number of patient studies has grown, because of the overall demand coming from the referring physicians, so has the need for reliable and reproducible imaged-derived parameters 
increased. For example, it has become a fundamental necessity to improve imaging reproducibility to allow comparison of data in the same patient over time for follow up studies performed either in the same or in different clinical centres.

In this perspective we have to read the work from Gazzilli et al who retrospectively assessed 108 patients with suspected IE, 54 of which were eventually diagnosed as such, who underwent $\left[{ }^{18}\right.$ F]FDG PET/CT from 2016 to 2020 . All patients were properly prepared for the scan with a low carbohydrate, high protein and high fat diet to reduce the high physiologic myocardial $\left[{ }^{18}\right.$ F]FDG uptake. In this study the authors try to overcome the simple imaging visual description by proposing a semiquantitative 4-point score, similar to Deauville score used in lymphoma, to estimate the performance of $\left[{ }^{18} \mathrm{~F}\right] \mathrm{FDG}$ PET/CT in detecting IE. The sensitivity, specificity, positive predictive value, negative predictive value, and accuracy of their score were 93\% [95\% confidence interval, CI 83\%-98\%), $81 \%$ (95\% CI 67\%-90\%), 84\% (95\% CI 75\%-9\%), $91 \%$ (95\% CI $80 \%-96 \%$ ) and $87 \%$ (95\% CI $79 \%-93 \%$ ), respectively. The study is a relatively small retrospective single centre trial. However, the number of patients included as well as the specific results achieved in this study are not what drove writing this editorial. Indeed, the scope of this editorial is to underline the importance of research on the topic that the above mentioned manuscript touched upon: the search for (semi)quantitative imaging parameters, commonly identified as imaging biomarkers, which will allow reproducible assessments and standardisation of imaging data analysis methods. As automation technologies such as machine-learning play an increasing important role in our everyday life, their potential effect on interpretation of imaging studies, unsurprisingly, becomes a major focus of research. ${ }^{6}$

After a time where we had to fight for, and demonstrate how valuable the application of a multimodality and multidisciplinary approach can be in the field of assessing IE, we are now at a second, not less important, turning point, when we need to grow and gain scale, providing prognostic evidence of how evolving technologies and workflows can benefit patients suffering from this highly fatal disease by positively affecting their outcome.

\section{Disclosure}

Paola Anna Erba and Ora Israel report no conflicts of interest related to this manuscript.

\section{References}

1. Habib G, Lancellotti P, Antunes MJ, Bongiorni MG, Casalta JP, Del Zotti F, et al. 2015 ESC Guidelines for the management of infective endocarditis: The Task Force for the Management of Infective Endocarditis of the European Society of Cardiology (ESC). Endorsed by: European Association for Cardio-Thoracic Surgery (EACTS), the European Association of Nuclear Medicine (EANM). Eur Heart J 2015;36:3075-128. https://doi.org/10.1093/eurheartj/eh v319.

2. Otto CM, Nishimura RA, Bonow RO, Carabello BA, Erwin JP III, Gentile F, et al. 2020 ACC/AHA guideline for the management of patients with valvular heart disease: A Report of the American College of Cardiology/American Heart Association Joint Committee on Clinical Practice Guidelines. Circulation 2021;143:e72-227. https://doi.org/10.1161/CIR.0000000000000923.

3. Erba PA, Lancellotti P, Vilacosta I, Gaemperli O, Rouzet F, Hacker $\mathrm{M}$, et al. Recommendations on nuclear and multimodality imaging in IE and CIED infections. Eur $\mathrm{J}$ Nucl Med Mol Imaging 2018;45:1795-815. https://doi.org/10.1007/s00259-018-4025-0

4. Slart RHJA, Glaudemans AWJM, Gheysens O, Lubberink M, Kero T, Dweck MR, et al. 4Is Cardiovascular Imaging: A joint initiative of the European Association of Cardiovascular Imaging (EACVI); European Association of Nuclear Medicine (EANM). Procedural recommendations of cardiac PET/CT imaging: standardization in inflammatory-, infective-, infiltrative-, and innervation (4Is)-related cardiovascular diseases: a joint collaboration of the EACVI and the EANM. Eur J Nucl Med Mol Imaging 2021;48:1016-39. https://doi. org/10.1007/s00259-020-05066-5.

5. Erba PA, Pizzi MN, Roque A, Salaun E, Lancellotti P, Tornos P, et al. Multimodality imaging in infective endocarditis: An Imaging Team within the Endocarditis Team. Circulation 2019;140:1753-65. https://doi.org/10.1161/CIRCULATIONAHA.119.040228.

6. Slart RHJA, Williams MC, Juarez-Orozco LE, Rischpler C, Dweck MR, Glaudemans AWJM, et al. Position paper of the EACVI and EANM on artificial intelligence applications in multimodality cardiovascular imaging using SPECT/CT, PET/CT, and cardiac CT. Eur J Nucl Med Mol Imaging 2021;48:1399-413. https://doi.org/10. 1007/s00259-021-05341-z.

Publisher's Note Springer Nature remains neutral with regard to jurisdictional claims in published maps and institutional affiliations. 